\title{
Estrategias docentes un reto actual en la educación
}

Alan Casasola Vázquez
Karen Victoria López López
Ilse Aglaee Waldo Gómez
Asesor: D.E. Esther Fragoso Fernández

Introducción

La presente investigación tiene como objetivo el análisis de las estrategias de enseñanza en docentes de la comunidad de Ayahualulco, se busca informar acerca del impacto que tiene la implementación de estas mismas en el aula. La información se recabó mediante la visita al lugar de estudio y se fundamentó con diferente literatura.

La presentación está dividida en los siguientes apartados: sustentos teóricos donde se fundamenta teóricamente la investigación. Metodología para describir el proceso por el cual se llevó a cabo la investigación. En el apartado de resultados se verá reflejada la información que se recabó mediante la aplicación de los instrumentos y los logros y conclusiones a los que éstos nos llevaron.

\section{Planteamiento del problema}

Esta investigación se derivó de la materia de Investigación Cualitativa, dado que para este tipo de experimentación es necesario un contexto donde se pueden realizar diferentes investigación de acuerdo a los intereses mostrados, podemos decir que la comunidad de Ayahualulco nos permitió realizar de manera pertinente la investigación sobre las diferentes habilidades docentes, la comunidad se encuentra situada en el estado de Veracruz, con un índice de población de 23 431 habitantes, de los cuales 11665 son hombres y 11766 son mujeres.

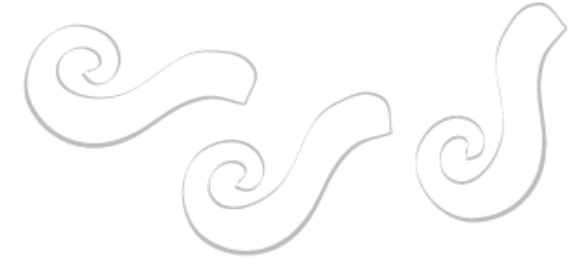

Dentro de esta comunidad se pretendió identificar las diferencias en las estrategias de enseñanza implementadas por el profesorado del nivel medio superior de esta localidad, la cual fue comparada con la escuela de los Hermanos Lasallistas de la comunidad de Ayahualulco, Veracruz.

Se buscó conocer el impacto que generan éstas en la adquisición de conocimiento en los alumnos de la escuela preparatoria de la comunidad, comparándola con la de los Hermanos Lasallistas, verificando los beneficios que tiene unas con otras, para así poder mejorar el nivel de cada uno de los alumnos.

\section{Preguntas de investigación}

¿Cuál es la diferencia entre las estrategias de enseñanza aprendizaje utilizadas en las diferentes escuelas de la comunidad de Ayahualulco?

¿Influye la capacitación del profesor en torno a las habilidades docentes, para su desenvolvimiento en el aula?

¿Cómo influye el número de alumnos en las aulas para la implementación de variadas estrategias de enseñanza?

\section{Objetivo General}

Conocer cómo los docentes utilizan las estrategias de enseñanza dentro del aula.

1 Sexto semestre de la Licenciatura en Ciencias de la Educación.(lauz19cas@hotmail.com)

2 Sexto semestre de la Licenciatura en Ciencias de la Educación. (sonrricks_69@hotmail.com)

3 Sexto semestre de la Licenciatura en Ciencias de la Educación.(ilse_waldo@hotmail.com) 

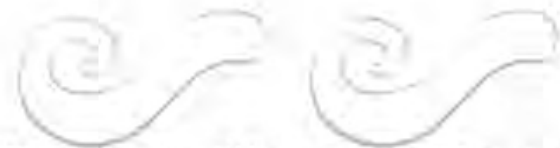

Conocer la diferencia en las estrategias didácticas implementadas dentro de las distintas escuelas de la comunidad de Ayahualulco.

\section{Objetivos específicos}

Observar el escenario didáctico y proponer actividades que ayuden al desarrollo de habilidades docentes.

Conocer cómo es la implementación de estrategias de enseñanza- aprendizaje dentro del aula en las escuelas de Ayahualulco.

\section{Justificación}

La comunidad de Ayahualulco se encuentra ubicada en la zona centro del estado de Veracruz; en la parte norte limita con Perote, en el noroeste con Xico, en el este con Ixhuacán de los Reyes y en el sur limita con el estado de Puebla. La investigación será desarrollada en una de las rancherías que se encuentran dentro del municipio de Ayahualulco.

La interrogante de la presente investigación se fundamentó en las necesidades planteadas en el proyecto PERLA que es definido como: "un proyecto unificador de criterios para la acción de las diferentes comunidades educativas; como un marco de referencia común para nuestros Distritos para el desarrollo del ministerio educativo Lasallista en la región; como un espacio privilegiado para la interdependencia; y como respuesta articuladora a las urgencias educativas" (La Salle, 2010).

Se analizaron las necesidades que maneja dicho proyecto, se decidió remitirse a la necesidad "democratización del conocimiento", la cual tiene como fin "Reelaborar, desde la comunidad educativa, el proyecto educativo a la luz del carisma fundacional, para orientarlo a la innovación y a la transformación social, tomando en consideración las necesidades de los más desfavorecidos y del contexto para ayudar y asegurar su acceso a la educación" (La Salle , 2010).

Orientándose en dicha necesidad, la presente investigación se dirige al ámbito educativo, enfocándose principalmente en las estrategias de enseñanza.

Es por esto que surge el interés por el estudio de las estrategias de enseñanza-aprendizaje, que son utilizadas en las aulas de las escuelas en Ayahualulco. Conocer la didáctica y los recursos utilizados en clase es importante para los futuros educadores ya que así, como hay escuelas que cuentan con los suficientes recursos tecnológicos, hay otras donde las carencias pueden ser un impedimento para la formación integral de los alumnos.

Según datos del INEGI el porcentaje de la población de 6 a 12 años que asiste a la escuela según sexo en el año 2000 y 2005 son los siguientes:

\begin{tabular}{|c|c|c|c|c|c|}
\hline \multicolumn{6}{|c|}{ Estado de Veracruz } \\
\hline \multicolumn{3}{|c|}{ Año 2000} & \multicolumn{3}{|c|}{ Año 2005} \\
\hline Total & Hombres & Mujeres & Total & Hombres & Mujeres \\
\hline 92.3 & 92.4 & 92.3 & 95.7 & 95.7 & 95.7 \\
\hline
\end{tabular}

INEGI 2010

Nuestro país atraviesa, desde hace algún tiempo, por un bajo nivel educativo, el cual se ve reflejado en su población, la educación se ha vuelto una necesidad primordial en la actualidad, tal vez si sabemos utilizar la educación en beneficio de nuestro país se puede hacer de él un lugar mejor, desapareciendo muchos de los problemas con los que se despierta cada día. La investigación se centró en el estudio de las habilidades docentes que ejecutan profesores de escuelas rurales así como la de los hermanos Lasallistas. Con esto se pretendió conocer, analizar y valorar las actividades que un docente desempeña frente a grupo, puesto que el proceso de enseñanza-aprendizaje influye 
mucho en la aplicación de estas durante el periodo de clase. Además se pretendió proponer una guía de actividades que le permitan al docente reflexionar y modificar ciertos hábitos de su actuar para con ello conocer o ampliar sus habilidades docentes.

\section{Viabilidad}

El principal recurso fue el humano ya que para esta investigación este personal consta de tres personas para su realización, el investigador uno cuenta con las capacidades de facilidad de palabra y adaptación al medio y a las condiciones del lugar, las debilidades de este son: interpretación objetiva de los datos y la investigación teórica, el investigador dos cuenta con la facilidad de convivencia y recolección de datos además de poder interactuar con todo tipo de gente, sus debilidades son la adaptación al medio y a las condiciones del contexto, el investigador tres cuenta con la facilidad de adaptarse al medio donde se encuentre, además de poder interpretar objetivamente los resultados de las encuestas realizadas, sus debilidades son la comunicación con las personas y lograr una convivencia con estos. El transporte es proporcionado por la escuela lo cual no presenta ningún problema para la realización de esta investigación, para la cuestión de tiempo todavía se nos presentan ciertas interrogantes ya que aún no sabemos específicamente cuántas escuelas encontramos en la localidad. Las habilidades con las que se cuentan para esta investigación se encuentran evaluadas por la acreditación de la materia y varias sesiones de indagación en las fortalezas y debilidades que se hallan en cada uno de los integrantes, además de que se realizaron diferentes prácticas en intervenciones en grupo, las planeaciones fueron revisadas con anterioridad por la docente encargada. En cuanto al tiempo se estima que en una semana se puedan alcanzar los objetivos propuestos.
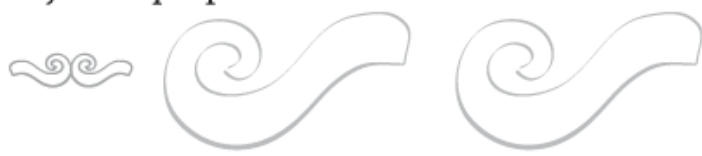

\section{Marcos}

\section{Marco Histórico}

En Mesoamérica, el centro educativo primordial (sino es que el único) era la familia. Los padres se encargaban de la instrucción de los niños, dándoles conocimientos sobre los oficios que se realizaban como la agricultura o la ganadería; mientras que las madres educaban a las niñas en las labores domésticas y las formaban para ser amas de casa. Después se formaron algunas instituciones, pero de igual manera a los niños se les instruía para el campo y las niñas para el hogar. "Algo diferente era la situación hace tres o cuatro siglos, cuando la educación se basaba en principios morales y normas de comportamiento, y cuando la asistencia a las escuelas sólo era accesible a grupos minoritarios" (INAFED, 2010). Es aquí cuando aparecen las divisiones en el acceso de la escuela, pues sólo podían acudir hombres que fueran de un alto estatus social.

Por ejemplo, las escuelas del mundo náhuatl era el Tepochcalli o el Calmécac, el ingreso a éstas era de los 3 a 9 años, a los 15 años. En el Tepochcalli se les instruía en los quehaceres cotidianos del campo y, como parte de su instrucción, participaban en la construcción de obras públicas tales como templos y obras hidráulicas. También se enseñaba civismo, empleo de las armas, artes y oficios, historia y tradiciones, así como la obediencia a las normas religiosas comunes. Los alumnos salían convertidos en guerreros.

Dentro del Calmécac se estudiaban las artes y las ciencias. La enseñanza era fundamentalmente religiosa, cuando muchos de los estudiantes no pretendían ser sacerdotes; algunos se preparaban para ocupar puestos en la milicia o la administración pública. El Calmécac era parte del Templo Mayor y los estudiantes estaban sujetos a una rigurosa disciplina. Les enseñaban a hablar bien, formación en astronomía y matemáticas.
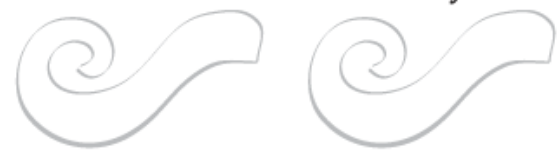

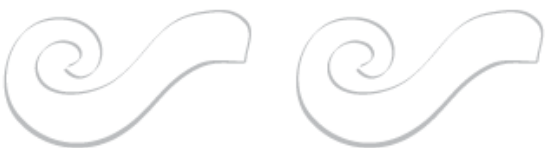

Estos son algunos ejemplos de las primeras escuelas que se establecieron.

Aun cuando ya existían diferentes textos literarios; cerca de la década de 1940 aparecieron diferentes libros y escritos relacionados específicamente con el campo de la educación su producción deriva de diversos investigadores, entre los que se reconoce a Max Miñano en 1945, Francisco Larroyo en el año 1947, Paula Alegría en 1963, José Luis Becerra en 1963, Josefina Vázquez 1970; Guadalupe Monroy en 1975, Guillermo de las Peñas y Luz Elena Galván en 1978, entre muchos otros; todos estos autores dirigen sus textos a la educación en cada respectivo año.

El perfeccionamiento del Sistema Nacional de Educación, es uno de los objetivos importantes para el óptimo desarrollo de nuestra sociedad. Una enseñanza que aspire a ser de calidad no puede desvincularse de las exigencias sociales. La formación de hombres integralmente desarrollados, que apliquen en la práctica los conocimientos asimilados, que sepan solucionar problemas, actúen creadoramente y posean los más elevados valores humanos, demuestra la calidad de cualquier sistema educacional. La educación en México es un derecho consagrado por la Constitución vigente, que en su artículo $3^{\circ}$ declara que la educación impartida por el Estado debe ser gratuita, laica y obligatoria para todos los habitantes del país. La Ley General de Educación obliga a quienes residen en México a cursar por lo menos los niveles primario y secundario de la educación, y establece que los jefes de familia tienen la responsabilidad de verificar que sus hijos cumplan con este deber. La ciencia y la tecnología han contribuido al desarrollo de los países y se ha convertido en un factor estratégico de supervivencia e integridad de las naciones.

Para esto México no está exento, ya que las economías globales de la era moderna exigen que los países vayan incrementando el uso de la ciencia y la tecnología y ésta a su vez debe
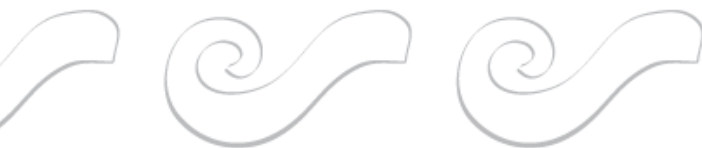

ser incorporada a la educación en todos sus niveles, México no puede quedar rezagado y para esto debe invertir en crear profesionistas que manejen las innovaciones tecnológicas para el beneficio de la sociedad y del país mismo.

Refiriéndonos específicamente al municipio de Ayahualulco se pueden identificar las siguientes escuelas:

- El Colegio Belisario Domínguez es una Escuela de primaria situada en la localidad de Ayahualulco. Imparte educación básica (primaria general), y es de control público (estatal). Las clases se imparten en horario Matutino.

- El Colegio Emmanuel Kant es una Escuela de Preescolar situada en la localidad de Ayahualulco. Imparte Educación Básica (Preescolar General), y es de control Público (Federal Transferido). Las clases se imparten en horario Matutino.

- El Colegio Ignacio De La Llave es una Escuela de Secundaria situada en la localidad de Ayahualulco. Imparte Educación Básica (Telesecundaria), y es de control Público (Federal Transferido). Las clases se imparten en horario Matutino.

- El Colegio Benito Juárez García es una Escuela de Primaria situada en la localidad de Ayahualulco. Imparte Educación Básica (Primaria General), y es de control Público (Estatal). Las clases se imparten en horario Matutino.

- El Colegio Federico Froebel es una Escuela de Preescolar situada en la localidad de Ayahualulco. Imparte Educación Básica (Preescolar General), y es de control Público (Estatal). Las clases se imparten en horario Matutino.

- El Colegio Sebastián Lerdo De Tejada es una Escuela de Primaria situada en la localidad de Ayahualulco. Imparte Educación Básica (Primaria General), y es de control Público (Estatal). Las clases se imparten en horario Matutino. 
- El Colegio Centro De Bachillerato Tecnológico La Salle es una Escuela de Bachillerato situada en la localidad de Ayahualulco. Imparte Educación Media Superior (Bachillerato Técnico), y es de control Privado (Particular). Las clases se imparten en horario.

\section{Marco conceptual}

Comunidad rural: Son aquellos sectores de la población que viven en condiciones donde los servicios como agua, pavimentación, alumbrado, etc. son escasos y aún utilizan métodos básicos para cubrir sus necesidades. Son las zonas marginadas donde la gente es humilde: el campo, sierras y algunos pueblos donde las costumbres antiguas son el estilo de vida que han adoptado por su poco contacto con la ciencia y tecnología. (Colmena Urbana, 2009).

Para esta investigación el concepto de comunidad rural será contemplado como un lugar donde las condiciones de la población se ven limitadas por la falta de servicios de primera necesidad.

Docente: Es la persona que enseña una determinada ciencia o arte. Es aquel que se le reconoce una habilidad en la materia que imparte. Debe poseer habilidades pedagógicas para convertirse en agente efectivo del proceso de aprendizaje. (http://definicion.de/docente, 2008). Para esta investigación se considerara al docente como la persona que cuenta con algunos conocimientos específicos los cuales le pueden ayudar a transmitir conocimientos, en un proceso de enseñanza-aprendizaje.

Habilidades docentes: Son las capacidades que permite al docente promover en su labor un aprendizaje activo y significativo, además de motivación, mejorar la comunicación y relaciones afectivas. (ITESM, 2006). Dentro de esta investigación se tomará a las habilidades como las capacidades que desarrolla el
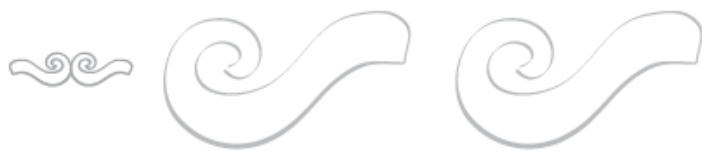

docente para ayudar a facilitar el proceso de aprendizaje.

Escuela primaria: Escuela en la que se enseña a leer y escribir, junto con los conocimientos básicos de la historia, la geografía, la naturaleza y el comportamiento cívico; lugar o edificio en donde se realiza: estudiar primaria, maestra de primaria, ir en primaria, hacer la primaria, certificado de primaria. (Sensagent, 2010) podemos considerar el siguiente concepto para la investigación: lugar en el cual se pretende transmitir conocimientos esenciales, como el leer y escribir, donde además se fomenta el amor por los símbolos patrios, contando con materias adicionales que ayudan al conocimiento de la geografía, historia y esencialmente las matemáticas.

Didáctica: Proviene del griego didasko y hace referencia a guiar y mediatizar los conocimientos y principios, con la instrucción, actividades prácticas y desarrollo de actividades, en todos los ámbitos: familiar, escolar, empresarial y comunitario.(Villalobos, 2010).

Para esta investigación la didáctica estará considerada como las habilidades y estrategias que implementa el docente para realizar las actividades dentro del contexto educativo, las cuales implican al alumnado y los padres de familia.

\section{Marco teórico}

La escuela no es una entidad aislada de la realidad social, muy por el contrario, tal como señala Savater, la escuela es la primera multiplicadora de intereses culturales a través de la transmisión de los conocimientos tecnológicos y de los valores que sustentan la convivencia social. Sin lugar a dudas es imposible negar que la institución es aquella fuente de conocimientos que prepara a la sociedad, tomando en cuenta sus condiciones, recursos, y necesidades que les permita sobrevivir, por eso es de suma importancia
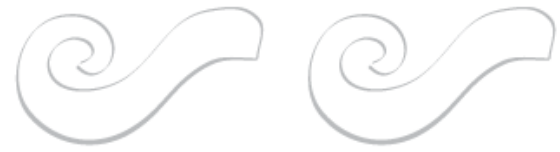

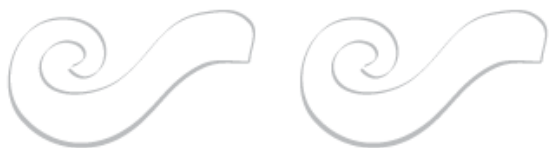

apoyar al sector educativo, para que guíe y conduzca a sus educandos a una formación integral y con sustentos teóricos.

Ya que pensar en un proceso es su totalidad implica no dejar las cosas a la improvisación, ni proceder a una mera adición de partes según se vayan presentando.(Zabalza, 2003)

En lo que se refiere a la planificación del proceso enseñanza-aprendizaje, es necesario siempre tener en cuenta las condiciones que rodean a la escuela. Más aun, según propone Pérez (1999:91) es tarea del Estado y de la sociedad unir esfuerzos para compensar las ausencias y desventajas estructurales de las escuelas insertas en comunidades de menores recursos proveyéndoles de bibliotecas, comedores escolares, salas de computación, canchas deportivas, lugares para investigar, actividades extra escolares atractivas, entre otros. Se trata de superar el fracaso y evitar la exclusión, así como de fortalecer los ambientes escolares que posean condiciones adecuadas para atender las necesidades de los alumnos que se benefician de ellos. Ya que acercando estos recursos, quizá no se garanticen buenos resultados, pero sí un avance, por lo menos de la sociedad en la que se realizó la investigación.

Así mismo, la Reforma Educativa en México menciona "la inclusión de la familia y la comunidad en los procesos de enseñanzaaprendizaje". (reformaeducativa.edu/México). En este sentido, se puede obtener un doble beneficio: en primer lugar la integración del muchacho en el medio social que le rodea fomentando los valores de participación ciudadana y democracia tal como se conciben en el marco jurídico que sustenta el Currículum Básico Nacional; en segundo lugar, el beneficio a la comunidad en función de los cambios que puedan generarse desdelas actividades escolares y los cambios estructurales que se lleven a cabo en la escuela. Vygotsky, ha investigado y descubierto leyes
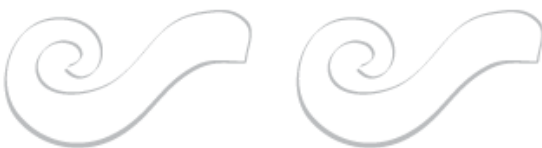

que rigen el desarrollo de habilidades. Estas investigaciones han demostrado que "las habilidades se desarrollan a través de la "apropiación de la cultura" por el sujeto en la dinámica de la actividad práctica con las personas y los objetos y en la comunicación entre los sujetos". Son estos los principios teóricos que orientan nuestra investigación. Lo que supone que la escuela es un agente socializador, y a través de ésta, se logra que una multiculturalidad que permite el desarrollo de la persona. (Hechavarría, 2010 en Morenza,1997).

"El perfeccionamiento del Sistema Nacional de Educación, es uno de los objetivos de nuestra sociedad". Una enseñanza que aspire a ser de calidad no puede desvincularse de las exigencias sociales. Ya que en la actualidad se suele confundir calidad con cantidad, y lo que se requiere es que la actividad docente plantee una enseñanza en el marco de la calidad, que introduzca los elementos necesarios en los educandos para que se enfrenten a nuevos retos y puedan solucionar situaciones dentro de su contexto social que abarque sus dimensiones de conocimiento.

Las estrategias didácticas para desarrollar habilidades profesionales en los futuros profesores deben considerar el papel del docente en el aprendizaje de los alumnos y las relaciones interactivas entre los estudiantes y de éstos con el profesor para solucionar los problemas del objeto de la profesión. Si no existe una vinculación de trabajo en equipo es imposible mejorar la práctica docente, ya que se deben tomar en cuenta las aportaciones del alumno así como las del docente y juntos encontrar nuevos métodos y estrategias para mejorar el proceso de enseñanza-aprendizaje. Lo que todos los profesores necesitamos es un conocimiento profesional de la docencia. Ése es nuestro trabajo y llevarlo a cabo en buenas condiciones exige saber de él, sobre su teoría y sobre su práctica, con una cierta profundidad. (Zabalza, 2003) 
“Un proceso para dirigir el desarrollo de habilidades profesionales, basado en el modo de actuación profesional, tiene la finalidad de enseñar a aprender y enseñar a pensar a los futuros profesores". Sin duda la finalidad sigue y seguirá siendo enseñar al alumno contenidos que modifiquen y lo guíen a ser una persona crítica y capaz de tomar decisiones que le permitan abrirse a nuevos paradigmas, para mejora de sus condiciones devida.

Para esto el docente deberá contar con ciertas competencias las cuales nos plantea Zabalza:

Competencias referidas a comportamientos profesionales y sociales: las cuales se refieren a la actuación del docente en cuanto a toma de decisiones, trabajo compartido y asunción de responsabilidades.

Competencias referidas a actitudes: tiene que ver con la forma en cómo afronta la relación con las personas: motivación personal, compromiso, trato a los demás y capacidad de adaptación.

Competencias referidas a capacidades creativas: cómo los sujetos abordan el trabajo en conjunto, si buscan soluciones nuevas, asumen riesgos, si son originales.

Competencias de actitudes existenciales y éticas: si es capaz de ver las consecuencias de las acciones profesionales, si posee un proyecto personal y fuerza para hacerlo realidad, si posee valores humanísticos $\mathrm{y}$ compromiso social.

El desarrollo de una habilidad dependerá de la forma que se organice el aprendizaje; de aquellos eslabones o procesos de la actividad que el sujeto sistematiza, del lugar que éstos ocupan en la estructura de la misma y en la conciencia del sujeto y del nivel de asimilación y dominio alcanzados. Para esto la propuesta didáctica para desarrollar habilidades profesionales nos da la oportunidad
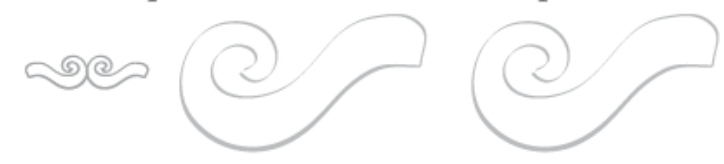

de entrar en la era del conocimiento, donde actualmente las cosas se renuevan cada día, lo que hoy es innovación mañana será obsoleto. "De la misma forma, opera con los medios educativos, de la evolución de la escuela como un medio físico a lo que hoy tenemos, salas virtuales y enseñanza a distancia" (http:/edu caciónporcompetencias.com/México).

El uso de las herramientas informáticas en la formación de profesionales es sin duda un instrumento básico en estos tiempos, es importante señalar cómo es que este cambio ha permitido una formación integral en los alumnos."Anteriormente se contaba sólo con las clases del tipo presencial, con el básico método de enseñanza-aprendizaje predicado por cientos de años, el cual llega sólo a un grupo de interesados en un momento y en un espacio". Y sin lugar a la inclusión de los medios de comunicación al ámbito educativo han venido a reformar la calidad en la educación, lo que supone que los alcances se acerquen más a los fines de la educación en México. (Artículos informativos, 2010)

\section{Marco referencial}

Para sustentar la investigación analizaremos los diferentes contextos en los cuales se desarrolla la mujer, dado que ésta se dirige aún más a este aspecto, empezaremos diciendo como bien lo mencionan en un artículo "una buena parte de la actividad comercial minorista de los mercados tradicionales de la zona es controlada por las mujeres" (Campbell \& Green, 1999), cosa que resulta importante, pues muchos de los hombres que habitaron este lugar migran para obtener mejores ganancias, por lo tanto las amas de casa, deben de buscar otra forma se obtener un ingreso extra, por lo que las mujeres deben de localizar nuevas formas de comerciar los productos de la región, además también se presentan con otro problema, dentro de este lugar se encuentran personas hablantes de lenguas indígenas, por lo cual se presenta un problema de extinción de la lengua, para

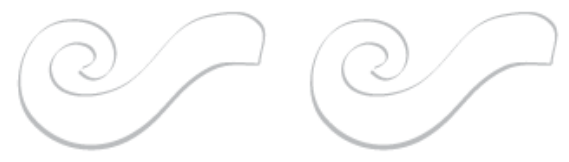

11 

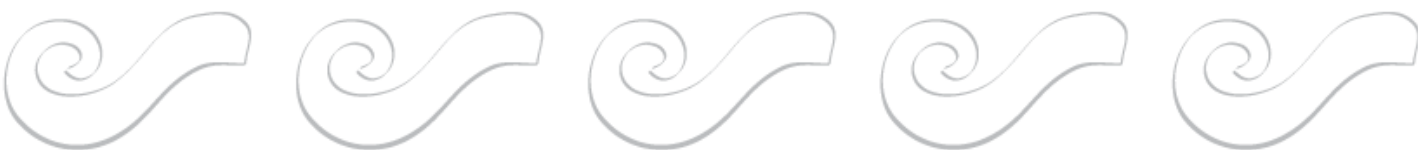

nosotros como investigadores no debe de presentar ningún problema.

Todos estos datos los pudieron recabar los investigadores después de analizar las descripciones hechas por los viajeros o aquellos que aparecen en pinturas, además de estudios antropológicos, diferentes escritos feministas además de comentarios hechos por los medios de comunicación.

Ahora empezaremos a situar más el papel de la mujer dentro de esta investigación, ya que dadas las circunstancias de la localidad donde se realiza la investigación, la mujer en la docencia dentro de la comunidad, juega un papel muy importante, "la práctica docente constituye una especial oportunidad para estos fines, ampliando su concepción y desarrollo más allá de la preparación y dictado de clases en las escuelas"(Morales, Redalyc, 2002), se le empieza a situar más dentro de esta investigación ya que la información que tenemos nos dice que los docentes que laboran en esta región son mujeres.

Este estudio se basa en estudios etnográficos tales como la entrevista no estructurada, observación participante $\mathrm{y}$, análisis de documentos escritos. El análisis contempló tres aspectos: las concepciones de las docentes antes de iniciar el proceso de actualización, el análisis del programa de actualización, las concepciones de las docentes luego de su participación en el programa.

Iniciaremos por analizar las diferentes estrategias que logran emplear dentro del aula, dado que estas son las bases para un mejor entendimiento de los contenidos dentro del aula, si bien "un problema mayúsculo dentro de nuestro sistema de educación se ve en las observaciones directas en escuelas oficiales nos han permitido constatar insuficiencias de dotación, gestión, funcionamiento y, especialmente, de atención a los procesos pedagógicos" (Rodríguez Trujillo, 2009).
Para el análisis de los resultados de la investigación citada anteriormente se utilizaron las notas de campo, actas de reuniones, informes, y un cuestionario, cuyo análisis cualitativo proporcionó evidencias para mejorar el procedimiento y los instrumentos utilizados. Las lecciones aprendidas se sintetizan en la configuración de un modelo de mejora profesional del docente.

El problema de la capacitación para los docentes en este tipo de zonas es que son de muy difícil acceso, además se presentan otras problemáticas con las que debe lidiar el docente, por ese mismo motivo se le ve como una persona flexible, ya que debe adecuar sus capacidades, para poder controlar las problemáticas de aprendizaje y así poder encontrar diferentes soluciones o aplicaciones especiales dentro del aula.

Dado que el análisis de esta investigación está basado en una metodología cualitativa, la cual se puede hacer a través de notas de campo, informes y cuestionarios, se realizó una interpretación de los resultados, la cual consistió en estructurar una codificación de estos para después, ordenarlos y verificar las diferencias que presentan cada una de estas. Ya que esto nos ayudara a visualizar la deficiencias por las cuales cruzan estas escuelas, debido a que su lejanía y problemas de accesibilidad ocasionan muchos problemas para los profesores, y para la misma comunidad. Para realizar estas mejoras también debemos de tomar en cuenta qué tan inmiscuida se encuentra la SEP (Secretaria de Educación Pública), dado que ellos deberían de ser los más interesados en la calidad de sus docentes sin importar el nivel en el que se encuentren laborando.

\section{Metodología}

\section{Enfoque:}

El enfoque usado para esta investigación es cualitativo. 
Alcance:

Exploratorio, ya que simplemente estamos interesados en saber cuáles son las problemáticas que se generan al no poder implementar las estrategias docentes.

\section{Diseño:}

El trabajo es no experimental debido a que la observación y aplicación de instrumentos se realizó en su contexto natural, sin realizar alguna alteración.

\section{Supuesto:}

Lo que se planteó dentro de este trabajo es la falta de capacitación de los profesores, por lo cual la implementación de las estrategias docentes se les dificulta, en algunos casos por el número de alumnos con el que trabajan los docentes dellugar.

\section{Contexto:}

La comunidad de Ayahualulco se encuentra ubicada en la zona centro del estado de Veracruz; en la parte norte limita con Perote, en el noroeste con Xico, en el este con Ixhuacán de los Reyes y en el sur limita con el Estado de Puebla.

\section{Población:}

La presente investigación se hizo en instituciones del municipio de Ayahualulco. Se realizó un análisis entre las estrategias utilizadas dentro del aula en las escuelas rurales y la escuela de los Hermanos Lasallistas. En ambas escuelas se atienden a los niños que cuentan con escasos recursos y poco acceso a la educación. La escuela de los Hermanos Lasallistas es la única en la región que brinda la educación media superior, las otras escuelas a analizar serán de nivel básico.

Para dicho análisis se tomaron como muestra las instituciones de las comunidades que están en la periferia de la Escuela de los Hermanos Lasallistas.

\section{Sujeto:}

El sujeto a analizar es el docente, se pretende hacer una diferenciación entre las estrategias
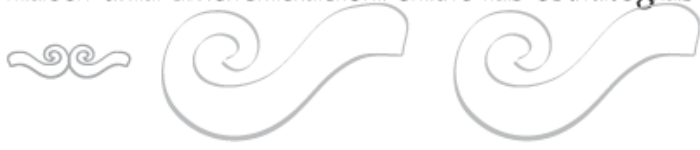

utilizadas que son utilizadas en el sistema Lasallista y las que se utilizan en el sistema de escuelas rurales. Las aportaciones y disposición que tenga el docente para el beneficio de la investigación será pieza fundamental para el éxito de esta misma.

\section{Muestra:}

Profesores de la escuela La Salle Ayahualulco; así como docentes de los diferentes instituciones en las comunidades aledañas a la escuela antes mencionada. Debido al bajo número de profesores con los que cuentan ambas comunidades educativas, se tomarán en cuenta aquellos que deseen involucrarse en la investigación.

\section{Procedimiento:}

Para la aplicación de instrumentos se visitaron las diferentes escuelas de la comunidad, incluyendo la de los Hermanos Lasallistas, para lo que se tomaron los grupos más avanzados de cada una de ellas, para los cuestionarios de padres, se realizaron de manera aleatoria, ya que muchos de ellos se encontraban realizando actividades laborales o domésticas.

\section{Resultados}

Para esta investigación se utilizó un cuestionario para los padres de familia y maestros, una encuesta para los padres de familia con preguntas orientadas hacia la asistencia a reuniones escolares, el interés que muestran por las tareas que realizan, además de poder saber qué nivel educativo tiene cada una de ellos. Lo anterior nos facilitará saber qué ayuda les pueden prestar a sus hijos en cuestiones escolares como las tareas. Estas acciones no sólo se realizaron dentro de las instituciones educativas, sino que algunas de ellas se hicieron en casas algunas familias.

A los docentes se les pidió su historia de vida dentro de su contexto natural, adecuándonos a sus horarios, para muchos de ellos era más
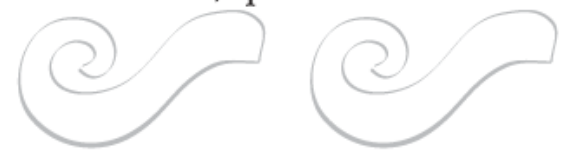

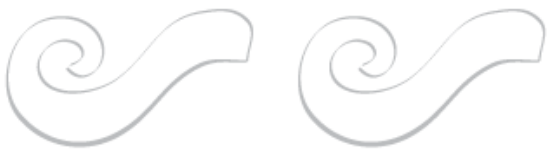

fácil ya que no todos viven dentro del poblado.

Después del análisis realizado a través de la interpretación, codificación, y tabulación de los resultados obtenidos dentro de los instrumentos de recolección de datos, se percibe que los seis profesores que tienen una mayor antigüedad que oscila entre los cinco años o más, no muestra una diferencia entre las actualizaciones que recibe, pues los profesores que tienen otro tipo de cursos o mayor grado de educación no muestran una diferencia con los profesores que sólo tienen una licenciatura, dado que únicamente asisten a tres actualizaciones al año. Con esto se puede decir que la antigüedad no es una factor para asistir a más talleres de actualización al año, además de que no es un factor muy notable el hecho que hayan asistido a una educación profesional en una universidad o una escuela normal, ya que el hecho de que tengan una formación pedagógica, no hace que sus recursos intelectuales hagan notoria su formación, pues el análisis muestra que los docentes se encuentran en una media con respecto a su formación.

En cuestión a la asistencia a talleres de actualización, sólo tres personas asisten a más de 5 talleres al año, pero la mayoría de profesores únicamente asisten entre 2 a 4 veces al año a este taller, y dos asisten sólo una vez, lo cual muestra que la asistencia a los talleres no genera mucha importancia por los mismos.

La utilización de las estrategias de enseñanza es aplicada dependiendo de la actividad planeada, pues cinco profesores creen que ese es el principal obstáculo para aplicarlas, debido a que no todos los niños pueden llevar el material extra que a veces solicitan los profesores. Otro factor en contra sería que muchos de los asistentes no van desayunados a la escuela, lo cual dificulta que presten atención a lo presentado por el profesor. Con
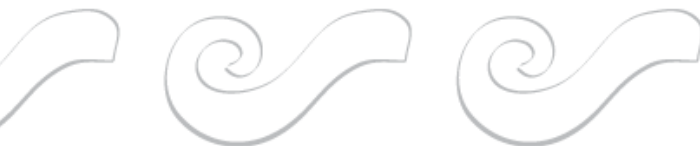

respecto al uso de la tecnología, no todos tienen acceso a ella y esto significa otro problema, además de que la experiencia demuestra que el número de alumnos no es la principal problemática al aplicar las estrategias docentes, y dos profesores dijeron que todo dependía de la asignatura, lo que puede ser un factor de cambio es la falta de experiencia, mientras que los tres restantes nos dicen que depende de otros factores como el tiempo destinado para la clase, las características particulares del grupo y los contenidos de la asignatura.

Para la aplicación de las estrategias se ve que para seis docentes el número de alumnos es una dificultad en la aplicación de los diferentes recursos, mientras que para los otros cuatro sólo en algunas ocasiones se les presentan dificultades.

$\mathrm{Al}$ analizar las dificultades por las cuáles las estrategias docentes no pueden ser aplicadas, surgen diferentes complicaciones, la principal es que cuatro personas consideran que los recursos financieros son uno de los principales problemas que tienen para enfrentar, mientras que en segundo lugar consideran que los recursos tecnológicos son otro obstáculo, ya que diferentes circunstancias dificultan tener acceso a este tipo de medios didácticos, y sólo dos personas dicen que las fallas en los sistemas de cómputo ocasionan otra dificultad que se presenta en la misma escuela lo cual hace notar que los recursos económicos no alcanzan para solucionar todos los problemas.

Los docentes nos dicen, a través de los cuestionarios, que la falta de tiempo es el principal problema con el que se enfrentan al desarrollar su práctica docente, y por consecuente se dificulta la aplicación de las diferentes estrategias docentes, al mismo tiempo dos de estos profesores subrayaron dos respuestas en las cuales se especifican que los recursos materiales también forman parte de sus prioridades, ya que el contexto no permite que los alumnos obtengan la mayoría 
de los materiales pedidos por éstos para trabajar dentro del aula, así mismo esto se aplica para la falta de espacios ya que dado el medio donde se encuentran las instituciones se dificulta que los padres de familia aporten recursos económicos de manera frecuente, pero se nota el apoyo que éstos tienen para con sus hijos al asistir de manera constante a las reuniones que se realizan en las escuelas.

Cuando empezamos a analizar los cuestionarios de los padres de familia nos damos cuenta que la mayoría de personas que nos contestaron son mujeres, solo dos hombres accedieron a contestar, así se puede ver que dentro de este contexto todavía las mujeres llevan la educación de los hijos, y los padres aún no se interesan en la educación de sus hijos, esto no significa que no pongan atención en que hacen y como lo hacen, sino que delegan esa responsabilidad a las madres de familia, además el compromiso con el cual se involucran las madres es importante ya que siempre están cuando las necesitan en la institución educativa.

Algo que facilita que ellas estén muy atentas a las solicitudes de los profesores es que se dedican a la atención del hogar, mientras que los hombres al ser proveedores del sustento económico o alimenticio del hogar solo se involucra en sus tiempos libres, lo cual no dice que no muestre interés sino que las actividad del trabajo no permite que él se encuentre al frente de la atención educativa.

Así mismo el interés que muestran las madres a las tareas que realizan los alumnos es muy alta ya que al decir que diecisiete de las madres encuestadas comentan que les prestan atención a sus hijos mientras que sus posibilidades les permitan orientarlos de acuerdo a los conocimientos particulares de cada madre, pues sólo unas pocas de ellas llegaron a cursar una educación a nivel medio superior, y sólo una de las madres nos dice que le presentan ciertas complicaciones para dedicarle mayor atención a su hijo que está en el kínder.
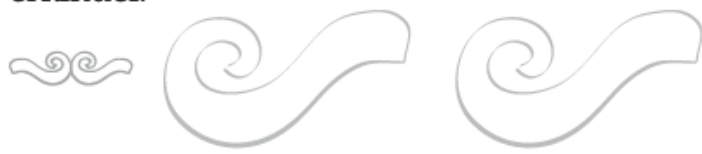

Para evaluar el rendimiento escolar de sus hijos las madres evalúan diferentes aspectos dependiendo de cómo sea la relación con los hijos, el profesor y las actividades diarias, así que al ver qué es lo que priorizan más las madres del municipio se hace notar que siete de ellas consideran que a través de las actividades diarias ellas notan el avance que tiene sus hijos en cuestiones educativas, por el contrario cinco de ellas consideran más importantes las calificaciones, otras parte de ellas se deja guiar por los comentarios de los profesores basándose en la realización de actividades, participación y cumplimiento de tareas, y la minoría se basa en los comentarios que les realizan los niños con relación a lo que hacen en la escuela y si se les facilita realizar las diferentes actividades.

Además de evaluar el rendimiento de sus hijos a través de diferentes rubros por parte de las madres, toman en cuenta la influencia de los profesores en los alumnos por lo cual ellas consideran que los maestros sí influyen en las calificaciones o el aprovechamiento de los alumnos, mientras que sólo dos dicen que los maestros no deben tener influencia en los alumnos.

Para las madres de la comunidad, la evaluación de los profesores se basa en las relaciones humanas; seis madres dicen que la forma en cómo interactúa el profesor con los alumnos se traduce en una forma de evaluación docente a los profesores, mientras que otras madres consideran que la facilidad con que los alumnos entienden las actividades es otra de las prioridades para evaluar a los profesores. En tercer lugar se encuentran las calificaciones con las que los alumnos son evaluados ya que esto refleja que se mezcla el entendimiento y las relaciones de los profesores con los alumnos, y para finalizar este análisis de la pregunta, se puede decir que las madres también se basan en el hecho de cómo los alumnos aprecian a su profesor y cómo se expresan de la forma en cómo los llega a tratar, pues para las madres de familia esto es importante y por eso verifican las relaciones quellevan durante el año escolar.

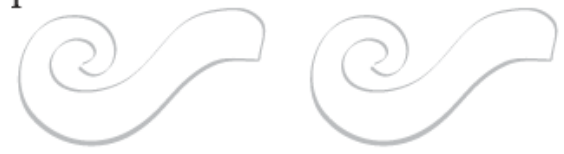



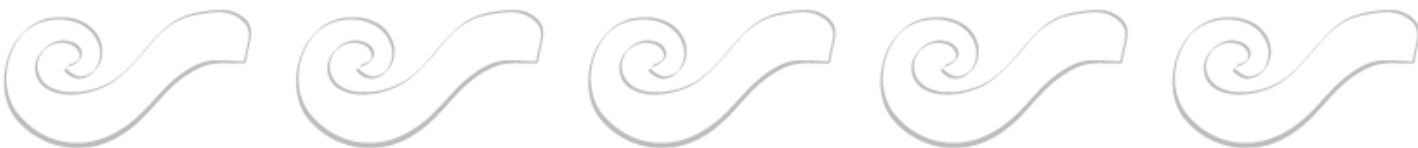

La secundaria de la Salle y las comunidades de Ayahualulco demuestran que la mayoría de sus alumnos asiste a clase en un porcentaje del $100 \%$ a asistencia. brincar esa instancia, pues la mayoría platica con él, y así intentan buscar una solución a la problemática que se les presente, la segunda opción es ir directamente con el directivo, pero lo que ellos nos dicen es que sólo llegan a esa instancia cuando después de una plática con el profesor, éste no realiza algún cambio, tienen que llegar hasta una instancia mayor que en este caso es el Director. Y una sola de ellas dice que no hace nada, porque considera que las cosas no pueden cambiar.

En las propuestas de mejora en la enseñanza 9 personas creen que a los profesores les hace falta mayor capacitación, pero también consideran que los profesores de mayor edad tienen más facilidades para controlar y enseñar a los alumnos, cuatro personas consideran que deberían de cambiar de profesores por personal más joven, ya que son sólo las personas que difieran de las que consideran que la experiencia es mejor, el argumento de estas personas es que estos profesores tienen mayores formas de enseñanza para sus hijos, y la misma cantidad dice que las formas de enseñanza pueden seguir igual, que no presentan problemas con las formas de enseñanza y sólo una persona considera que se deben fortalecer las relaciones alumno maestro, comprometiéndose los dos en cada una de las actividades que realicen.

Según los resultados arrojados por la guía de observación se puede afirmar que dentro del sistema educativo de la secundaria La Salle Ayahualulco los docentes asisten en su mayoría con puntualidad a su clase, contando con un porcentaje de $87.5 \%$ en afirmación a esta cuestión y en negación con $12.5 \%$. Así mismo el de las comunidades aledañas a Ayahualulco se cuenta con un $25 \%$ de asistencia puntual a las clases y un $75 \%$ de impuntualidad.
Durante la visita a las aulas de la secundaria $\mathrm{La}$ Salle se pudo observar que el docente utiliza material distinto al pizarrón y marcadores como material didáctico (cubos, figuras geométricas de tamaño grande, mapas y rotafolios) durante su práctica docente, contando con un $62.5 \%$ en cifras positivas y una diferencia de $37.5 \%$ negativas. Sin embargo, en un $87.5 \%$ el docente utiliza material distinto al pizarrón ya que tienen todo el salón adornado de los números en grande, del abecedario, muñecos, paisajes y figuras para complementar los temas y un $12.5 \%$ no lo utiliza. Esto en respecto a las escuelas de las comunidades de Ayahualulco.

Los resultados arrojan que el lenguaje entre maestro alumno es entendible en los dos tipos deinstituciones.

Se puede afirmar que el $62.5 \%$ de los docentes de la secundaria La Salle, atrae la atención de los alumnos y un $37.5 \%$ no lo logra este objetivo, porque les falta utilizar material de apoyo para complementar los contenidos, así como la realización de actividades que sean del interés de los alumnos. En contraste con los docentes de las escuelas de las rancherías, con su explicación atraen la atención del alumno en su totalidad porque ellos utilización de otros recursos materiales y actividades relacionadas con el contexto de los niños que despierta el interés en ellos.

Se demuestra que en un $62.5 \%$ los alumnos participan en clase y un $37.5 \%$ no lo hace. Referente a las instituciones de las comunidades aledañas a Ayahualulco se puede observar que en un $75 \%$ los alumnos participan activamente en clase y teniendo una diferencia negativa de $25 \%$, referente al anterior. 
Como resultado de lo observado se confirma que el docente aplica estrategias de integración grupal en un $75 \%$, como el trabajo en equipo, tareas en equipo, y actividades para fomentar el compañerismo. En cambio, en la aplicación de estrategias de integración grupal el $50 \%$ lo realiza y un $50 \%$ no lo efectúan, en las de los poblados.

Los resultados obtenidos en la guía de observación en la secundaria La Salle demuestran que en un $87.5 \%$ los alumnos realizan las actividades solicitadas por el docente y en un opuesto el $12.5 \%$ no lo hace.

El $87.5 \%$ de los alumnos que asisten a la secundaria La Salle como las de las rancherías realizan las actividades solicitadas por el docente y un $12.5 \%$ no lo hacen. Ya que esto se debe a que algunos alumnos no entienden las instrucciones o no tienen completamente dominados los conocimientos.

En el caso de las escuelas vecinas a Ayahualulco así como de la Salle se comprueba que en su totalidad, sus docentes están al pendiente de las dudas e inquietudes de sus alumnos.

La observación arroja que en un $50 \%$ de los docentes Lasallistas da un cierre o conclusiones a las clases. En las escuelas visitadas de los poblados cercanos a Ayahualulco, se puede observar solo que el 12.5 da un cierre o conclusiones a la clase y con una diferencia de 12.5 negativamente. Sin embargo en un $75 \%$ no se pudo concluir la observación hasta finalizar la clase, por lo que no se obtienen resultados significativos en cuanto a esta cuestión.

\section{Conclusiones}

La aplicación de instrumentos de historia de vida se realizó en la comunidad educativa de La Salle Ayahualulco y en diferentes instituciones de las comunidades aledañas a la escuela antes mencionada, contando con la participación de 9 docentes, 4 de las

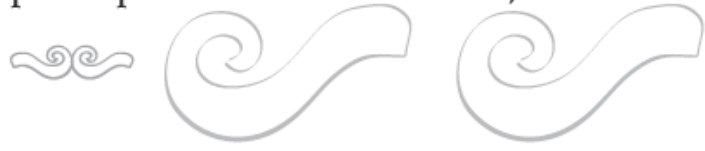

instituciones de las diferentes rancherías y 5 de la Secundaria La Salle.

Los resultados arrojados en esta aplicación se identifican como de los 5 maestros Lasallistas, 4 contestaron de manera acorde a lo que se les pidió, hablando en su mayoría de las actividades que han realizado en años escolares pasados, donde se hace uso de técnicas como la exposición, las representaciones, el café literario, la evaluación y coevaluación. El otro profesor expresó la actividad que realizan al final del ciclo escolar, la cual se basa en la exposición de lo aprendido, demostraciones del trabajo que se hizo durante el año, estas son tanto para alumnos como para padres de familia.

En cuanto a los profesores de las instituciones en las comunidades cercanas a La Salle Ayahualulco, las respuestas dadas pueden ser adaptadas a la investigación, pues se abordan temas como grupos grandes de alumnos, donde el rendimiento no es bueno y sin embargo el profesor actúa e interviene de manera adecuada para mejorar el promedio del grupo. También se abordan ideas sobre la falta de recursos tanto en las escuelas como en las casa de los alumnos, esto sin duda afectará el rendimiento académico, pero si el maestro busca las estrategias de las que puede hacer uso con los recursos con los que cuenta, podrá encaminar su proceso de enseñanza para generar un buen aprendizaje en los alumnos. Se eliminó la participación en este instrumento de un maestro, puesto que su respuesta no es acorde con la investigación, ya que habla acerca de los accidentes que pudieran ocurrir mientras los niños juegan, dada esta opinión, no se puede adaptar al tema de estrategias de enseñanza.

Para esto ayudándonos de los cuestionarios hechos a los padres de familia de las formas de enseñanza que plantean los profesores a sus hijos, todos ellos se interesan en la forma en que sus hijos son educados, para lo cual la asistencia de forma constante a las reuniones que realizan los profesores para facilitar la
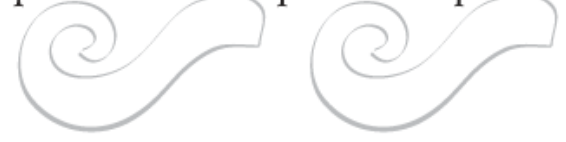

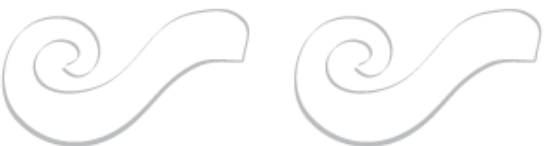

forma de enseñanza a los alumnos de ese lugar.

Se presenta una gran diferencia entre las estrategias que implementan los docentes de la comunidad de Ayahualulco y la de los profesores de La Salle, ya que muchos de los profesores de la comunidad se encuentran en gran desventaja, en los cuestionarios aplicados a cada uno de ellos estos nos hacen notar de manera muy sobresaliente que dentro La Salle la capacitación a cada uno de ellos es más constante y se adapta a las necesidades de los alumnos, lo cual en la comunidad es más difícil.

Para lo cual podemos mencionar que aunque algunos de los profesores de la comunidad se encuentran recién egresados de alguna licenciatura no presenta ninguna dificultad para los profesores de La Salle en aplicar las diferentes estrategias docentes, mientras que para los de la comunidad, la falta de experiencia es una complicación, y para la mayoría de personal docente de ambas instituciones la experiencia es un arma para desempeñar de manera más efectiva su labor, presentando para algunos más complicaciones, debido a la falta de la antes ya mencionada.

Los padres de familia consideran que a algunos de ellos les hace falta capacitación para enseñarles en una forma óptima a los alumnos, algunos de ellos no consideran muy bueno que lleguen profesores nuevos a las escuelas dela región.

Muchos de los profesores dicen que el hecho de que los alumnos no asisten con el material necesario para poder trabajar es una dificultad, pocos de ellos mencionan que el número de alumnos sea un problema, por lo tanto los problemas son más relacionados con el contexto por lo que un curso adicional sería una buena opción para mejorar la forma de enseñanza.
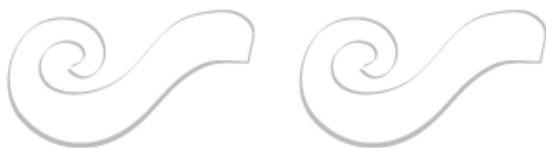

En relación al lenguaje entre maestro alumno se puede demostrar que en su totalidad es entendible, ya que el lenguaje que se utiliza esta adecuado al nivel de maduración y de edad que demanda las necesidades de los alumnos, propiciando la atención del alumno a la clase lo que genera que en su mayoría los alumnos participen activamente tanto en las rancherías como en la escuela La Salle.

A consecuencia de la asistencia en su mayoría de los alumnos a la institución a la institución, el docente puede aplicar estrategias de integración grupal en $75 \%$ con una diferencia de $25 \%$ negativamente en La Salle lo que propicia un ambiente tranquilo y cálido. Sin embargo a diferencia de esta, las de la ranchería demostraron que se trabaja menos en la integración grupal ya que los grupos son muy pequeños y muy poco equilibrados, pues no todos tienen los mismos conocimientos, generando así una barrera para la educación de manera igualitaria.

En el material que utiliza el docente en su intervención educativa se puede observar que utiliza material diferente al pizarrón y marcadores tales como material de apoyo, elementos gráficos y didácticos, estimula a los alumnos a realizar las actividades que solicita, así mismo está al pendiente de atender las dudas e inquietudes de los alumnos lasallistas durante la realización de las actividades, y en comparación con las rancherías, el material es más variado que el de La Salle, pero aun con esto también logra que los alumnos cumplan elmismo objetivo.

Como resultado la actitud positiva de los docentes en relación a que acuden en su gran mayoría puntualmente a clase, porque cumplen con los tiempos establecidos, origina que los alumnos asistan con frecuencia a la escuela y se puedan cumplir con los contenidos programados, lo mismo sucede en La Salle, aunque con un nivel de exigencia más alto, tanto a docentes como alumnos. 
En general, se cumplió el objetivo planteado, ya que se tuvo contacto con dos tipos de institución las de Rancherías y La Salle, lo que permitió conocer cómo los docentes aplican las estrategias de enseñanza. Sin embargo se tuvieron algunas dificultades, ya el tiempo no fue suficiente, y la disponibilidad de algunos docentes no era positiva, y un integrante del equipo no pudo adaptarse fácilmente al contexto. Aun así se logró obtener información significativa.

\section{Sugerencias}

Un curso para poder manejar las condiciones del lugar en cada una de sus clases, lo cual facilitaría la implementación de las estrategias docentes sin perjudicar la economía de cada una de las familias del lugar, la cual podrían desarrollar dentro de cada una de las instituciones, ya que tienen mayor conocimiento de las necesidades de cada uno de los alumnos. Esto facilitaría el conocimiento de cada uno de los profesores a los alumnos.

La segunda sugerencia sería una tutoría dentro de algún momento dentro de la escuela, ya que muchas de las dificultades de los alumnos son de otra índole, el hecho de la falta de dinero dentro de los diferentes hogares en diferentes cantidades es una causa muy recurrente, pero los problemas familiares también forman una dificultad lo cual puede distraer a los alumnos de sus actividades escolares.

La tercera sugerencia dirigida a escuelas de la comunidad, lo cual consiste en manejar materiales que los alumnos pueden encontrar en sus casas, así estimulan otros apartados de su creatividad, y ambos se ayudan a crear nuevos materiales didácticos, por lo tanto para esto la profesora que presenta esta nueva idea podría impartir el curso para todos los profesores de las comunidades aledañas.

Se sugiere a la escuela La Salle crear el grupo de tutores, que puedan atender y escuchar los problemas de los alumnos, y estar al pendiente de su desarrollo físico, emocional e intelectual. Crear talleres de asesoramiento para los docentes en cuanto a la realización de material didáctico, ya que se pudo observar que hace falta complementar los contenidos, y esto puede estimular a los alumnos a que aprendan con más facilidad.

Esta sugerencia es para las escuelas de los poblados, para que los docentes organicen y relacionen los contenidos con actividades que puedan desarrollar en su contexto los niños y a su vez les permita entender sus condiciones. Que no solo se impartan los contenidos dentro del aula.

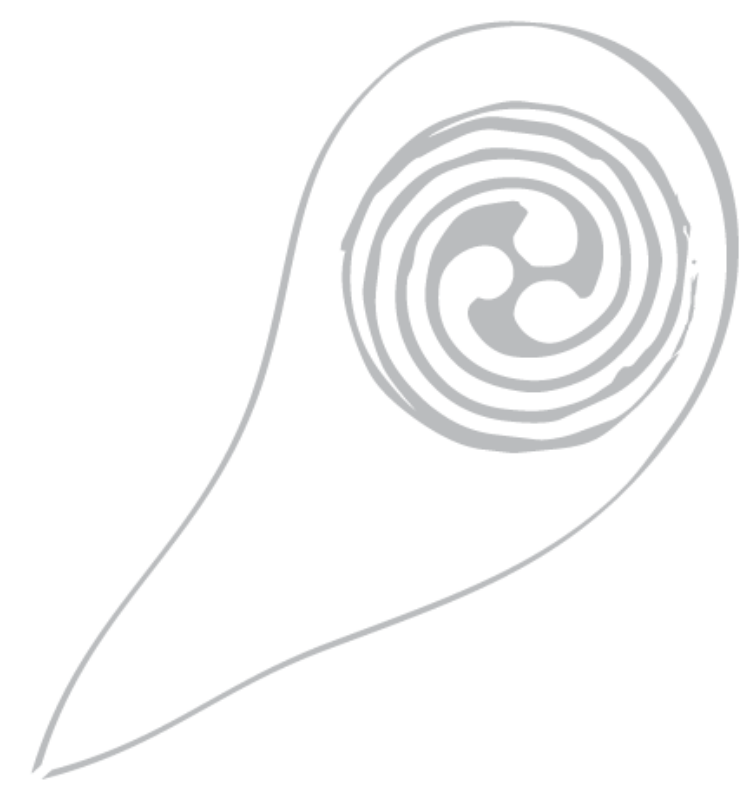




\section{Fuentes deconsulta:}

Articulos informativos (s.f.)Recuperado el 08 de 11 de 2010. En línea en: http://www.articulos informativos.com.mx/La_Evolucion_de_la_Ensenanza_Mexico-r1147419-Mexico.html

Campbell, H., \& Green, S. (1999). Redalyc.Recuperado el 08 de 11 de 2010, de Redalyc: En línea en: http://redalyc.uaemex.mx/redalyc/src/inicio/ArtPdfRed.jsp?iCve $=31600905$

Colmena urbana (2009). Recuperado el 15 de 11 de 2010, En línea en: http://colmenaurbana. blogspot.com/2009/01/el-concepto-de-lo-rural-y-lo-urbano.html

Definiciónes en linea (2008) Recuperado el 15 de 10 de 2010, En línea en: http://definicion. de/docente/

Elizondo, A. H. (2003). La nueva escuela, Dirección, liderazgo y getión escolar. México: Paidós.

Hechavarría, L. P. (s.f.). Monografias.com. Recuperado el 08 de 11 de 2010, En línea en: http://www.monografias.com/trabajos13/artestrg/artestrg.shtml

Inafed (2010). Recuperado el 10 de 09 de 2010, En línea en: http://www.inafed.gob.mx/ wb/ELOCAL/ELOC_Historia_de_la_Educacion

Inegi(2010). Recuperado el 07 de 08 de 2010, En línea en: http://www.inegi.org.mx/ est $/$ contenidos $/$ espanol $/ \mathrm{soc} / \mathrm{sis} / \mathrm{sisept} /$ default.aspx? $\mathrm{t}=$ medu37\&s $=\mathrm{est} \& \mathrm{c}=17034 \& \mathrm{e}=30$

Jurgenson, J. L. (2007). Cómo hacer investigación cualitativa. México, DF: Paidos Educador.

La Salle (2010). Recuperado el 10 de 08 de 2010, En línea en: www.lasallep.edu.mx

Morales, O. A. (2002)Redalyc. Recuperado el 15 de 10 de 2010. En línea en: http://redalyc.uaemex.mx/redalyc/src/inicio/ArtPdfRed.jsp?iCve $=35601911$

Rodríguez Trujillo, N (2009)Redalyc. Recuperado el 15 de 11 de 2010, En línea en: http://redalyc.uaemex.mx/redalyc/src/inicio/ArtPdfRed.jsp?iCve $=65911663007$

Sensagent. (2010). Recuperado el 15 de 10 de 2010, En línea en: http://diccionario. sensagent.com/primaria/es-es/

Villalobos, E. M. (2010)Didactica Integrativa y el Proceso de Aprendizaje.México: Trillas.

Valladares, C. (s.f.). Enciclopedia de los Municipios de México. Recuperado el 15 de09 de 2010, de Enciclopedia de los Municipios de México: http://www.inafed.gob.mx/work/ templates/enciclo/veracruz/municipios/30025a.htm

Zabalza, M. A. (2003)Competencias docentes del profesorado universitario . Madrid, España: Narcea, S.A
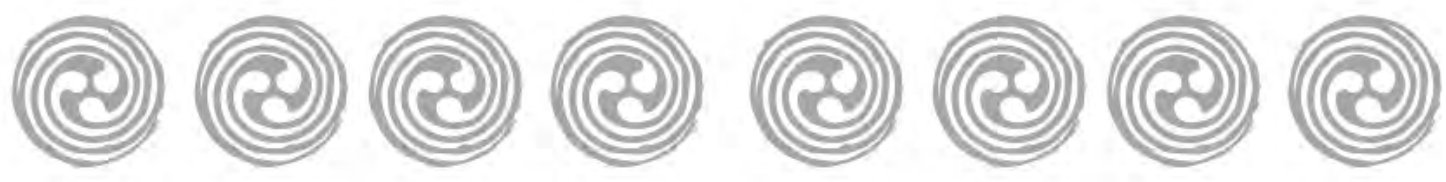